\title{
Gravitational memory of natural wormholes
}

L. A. Anchordoqui, M. L. Trobo, H. Vucetich, and F. Zyserman

Departamento de Física, Universidad Nacional de La Plata

CC. 67 (1900) La Plata

Argentina

\begin{abstract}
A traversable wormhole solution of general scalar-tensor field equations is presented. We have shown, after a numerical analysis for the behavior of the scalar field of Brans-Dicke theory, that the solution is completely singularityfree. Furthermore, the analysis of more general scalar field dependent coupling constants indicates that the gravitational memory phenomenon may play an important role in the fate of natural wormholes.
\end{abstract}

PACS number(s): 04.20.Gz, 04.50.+h 
Notwithstanding various possible exceptions, the mandate of the energy conditions (encoded in the evolution of the expansion scalar governed by Raychaudhuri's equation) is a popular tenet of belief in any current research field [1]. The simplest possible stellar environment that militate against these conjectures is usually called a "wormhole throat" [2].

Wormhole physics dates back at least as far as 1916 [3], with punctuated revivals of activity following both the classical work of Einstein-Rosen [4] and the seminal paper by Wheeler [5], but, the concept of traversable wormhole was not put forward until 1988 with the work by Morris-Thorne (MT) [6]. Nowadays, the broad phylum of wormholes can be precisely defined in terms of null geodesic congruences propagating outward from, and orthogonal to, an extremal hypersurface of minimal area known as the wormhole throat [7]. Unlike MT's embedding approach, this new characterization (that accurately captures the essence of the local physics which operates near any wormhole throat) is free from assumptions about symmetries, asymptotic flattness, embedded diagrams and even time dependence. We hasten to point out however, that throughout this article we shall use the word "wormhole" to specify the sub-class of traversable entities introduced by MT [6], namely, a compact $S^{2}$ tunnel connecting two asymptotically flat spacetimes. In order to expand such a configuration along the radial direction, the derivative of the $S^{2}$ radius with respect to the proper coordinate $l$ must be positive near the throat. Since this derivative is proportional to the energy momentum tensor on the radially directed null geodesic, a large inward pressure is required on the tunnel to sustain the wormhole structure, yielding a violation of the null energy condition.

Very recently the consequences of the energy conditions were confronted with possible values of the Hubble parameter and the gravitational redshifts of the oldest stars in the galactic halo [8]. It was deduced that for the currently favored values of $H_{0}$, the strong energy condition should have been violated sometime between the formation of the oldest stars and the present epoch. If also the null energy condition could have been violated and the universe does admit wormhole topologies in it, it is very likely that gravitational microlensing effects of these "exotic" objects could be monitored here on Earth [9]. The 
search for such a wormhole signature has been already started, but no conclusive evidence of wormhole topologies has been found neither in the galactic halo nor at cosmological distances [10].

On the other hand, wormhole formation at a late cosmic time requires a Lorentzian topology change, something that appears to be more than problematic to most physicists because it implies causality violations [11,12]. However, if wormholes are not created by astrophysical processes but were threaded in the embryo of the spacetime, one could expect a cosmological population of these objects without the uncomfortable predictions of topology change theorems. Building on this theme, Hochberg and Kephart proposed a subway network of wormholes, that might have arisen out during the quantum gravity epoch, as an alternative solution for the cosmological horizon problem [13].

Despite no universal mechanism to generate a relic density of exotic matter is well established yet (because of our ignorance of quantum gravity laws), if wormholes are leftovers from the primeval explosion, it is much more likely that their creation was accompanied by the extremely large curvature fluctuations generally believed to have existed during the Plank scale. For this reason, alternative theories of gravity have become a commonplace in wormhole physics. The already discussed scenarios are higher derivatives theories [14], non symmetric theories [15], Einstein-Gauss-Bonnet theory [16] and Brans-Dicke theory [17], among others. The latter actually is a special case of a more general group known as scalar-tensor theories (STTs) [18]. In such a class of theories (and also in the hyperextended version [19]) the equation of motion for test particles is identical to that of general relativity (GR), but the locally measurable value of the gravitational constant $G$ is a function of a scalar field which is in turn determined by the trace of the energy momentum tensor of all other non-gravitational fields. Recently, STTs have regained popularity in the understanding of the very early universe. On the one hand, it has been shown that these theories might drive new forms of inflation [20]. On the other hand, it has been suggested that an epoch dependent asymptotic boundary condition of the scalar field may have important implications in astrophysical objects such as black holes [21] or boson stars [22]: they may 
retain memory of the value of the gravitational constant at the time of formation, or evolve changing their configuration according to the variation of $G$. It, therefore, seems natural that in the context of wormhole physics this gravitational memory may play an important role. With this in mind, we shall search for a wormhole solution in general STTs, and after working out a theoretical construction of such a system we shall briefly discuss on its possible graviational memory [23]. Let us start by deriving the generalized field equations.

The fundamental action can be cast in the following form [24],

$$
S=\int \frac{\sqrt{-g}}{16 \pi} d^{4} x\left[\phi R-\frac{\omega(\phi)}{\phi} g^{\mu \nu} \phi_{, \mu} \phi_{, \nu}+16 \pi \mathcal{L}_{\mathrm{m}}\right],
$$

where $g_{\mu \nu}$ stands for the metric tensor, $g \equiv \operatorname{det} g_{\mu \nu}, R$ is the scalar curvature of the spacetime, $\phi$ is the scalar field, $\omega(\phi)$ is a dimensionless function which calibrates the coupling between the scalar field and gravity, and $\mathcal{L}_{\mathrm{m}}$ is the Lagrangian of the matter content of the system. Varying the action with respect to the dynamical variables $g_{\mu \nu}$ and $\phi$ yields the field equations

$$
\begin{gathered}
R_{\mu \nu}-\frac{1}{2} g_{\mu \nu} R=\frac{8 \pi G_{\infty}}{\phi} T_{\mu \nu}+\frac{\omega(\phi)}{\phi^{2}}\left(\phi_{; \mu} \phi_{; \nu}-\frac{1}{2} g_{\mu \nu} \phi^{; \alpha} \phi_{; \alpha}\right)+\frac{1}{\phi}\left(\phi_{; \mu \nu}-g_{\mu \nu} \square \phi\right), \\
\square \phi \equiv \phi_{; \alpha}^{; \alpha}=\frac{1}{2 \omega(\phi)+3}\left[8 \pi G_{\infty} T-\frac{d \omega}{d \phi} \phi^{; \alpha} \phi_{; \alpha}\right],
\end{gathered}
$$

where the Ricci tensor reads

$$
R_{\mu \nu} \equiv \Gamma_{\mu \nu, \alpha}^{\alpha}-\Gamma_{\mu \alpha, \nu}^{\alpha}+\Gamma_{\mu \nu}^{\alpha} \Gamma_{\alpha \beta}^{\beta}-\Gamma_{\mu \beta}^{\alpha} \Gamma_{\alpha \nu}^{\beta}
$$

with the Riemannian conection

$$
\Gamma_{\mu \nu}^{\alpha}=\frac{1}{2} g^{\alpha \beta}\left(g_{\beta \mu, \nu}+g_{\beta \nu, \mu}-g_{\mu \nu, \beta}\right) .
$$

We have introduced $T_{\mu \nu}$ as the energy momentum tensor of matter and all nongravitational fields, and $T$ as its trace. From now on we assume $T=0$ and we use "geometrized units" in which the speed of light and $G_{\infty}^{\text {today }}$ are equal to unity. Commas denote partial derivatives with respect to the coordinates $x^{\mu}$, semicolon mean covariant derivatives, and primes derivatives with respect to $r$. 
The theory can also be represented as Einstein's general relativity, with a new scalar field as an additional external non gravitational field that contributes to the curvature of the spacetime through its energy momentum tensor. This form of the field equations is derived from (2) and (3) by means of the conformal transformation $g_{\mu \nu} \equiv \bar{g}_{\mu \nu} \phi_{\infty} / \phi$ (in order to keep the next results as simple as possible we shall assume that $\phi_{\infty}=1$, and that $\phi$ in the following expressions is dimensionless). With the transformed metric $\bar{g}_{\mu \nu}$ we can define all required geometrical objects, namely, $\bar{R}_{\mu \nu}$ is defined as in (幽 using the metric $\bar{g}_{\mu \nu}$ in place of $g_{\mu \nu}$, or equivalently, we can compute the transformed Ricci scalar $\bar{R}$ (for details see, for instance, [25]). Substitution of the latter into the basic action (11), and the use of $\sqrt{-g} \equiv \phi^{-2} \sqrt{-\bar{g}}$, yields the action in the conformal representation

$$
S=\int \frac{\sqrt{-\bar{g}}}{16 \pi} d^{4} x\left[\bar{R}-\bar{g}^{\mu \nu}[\omega(\phi)+3 / 2] \frac{\phi_{, \mu} \phi_{, \nu}}{\phi^{2}}+3 \bar{g}^{\mu \nu}\left(\frac{\phi_{, \mu}}{\phi}\right)_{; \nu}+16 \pi \frac{\mathcal{L}_{\mathrm{m}}}{\phi^{2}}\right] .
$$

Hereafter we discard the third term in the action since it involves a total derivative. Redefining the scalar field by

$$
\varphi_{, \alpha}=|\omega(\phi)+3 / 2|^{1 / 2} \frac{\phi, \alpha}{\phi},
$$

one easily verifies that the action finally adopts the form

$$
S=\int \frac{\sqrt{-\bar{g}}}{16 \pi} d^{4} x\left[\bar{R}-\bar{g}^{\mu \nu} \varphi_{, \mu} \varphi_{, \nu}\right]+\bar{S}_{\mathrm{m}}
$$

where $\bar{S}_{\mathrm{m}}$ represents the conformal action for the matter fields. The field equations in the transformed frame read then,

$$
\square \varphi=0,
$$

and

$$
\bar{R}_{\mu \nu}-\frac{1}{2} \bar{g}_{\mu \nu} \bar{R}=8 \pi \bar{T}_{\mu \nu}+\left[\varphi_{; \mu} \varphi_{; \nu}-\frac{1}{2} \bar{g}_{\mu \nu} \bar{g}^{\alpha \beta} \varphi_{; \alpha} \varphi_{; \beta}\right]
$$

Let us introduce now the standard form for the metric tensor of a static spherically symmetric wormhole [6] 


$$
d s^{2}=-e^{2 \Phi(r)} d t^{2}+(1-b(r) / r)^{-1} d r^{2}+r^{2} d \Omega^{2}
$$

where $d \Omega^{2}$ stands for the line element of the sphere $S^{2}$. It contains two unknown functions: i) the redshift function which is assumed to be such that $e^{2 \Phi(r)}$ is never zero -this ensures the absence of an event horizon- and ii) the shape function $b(r)$.

Our choice for the redshift is fairly simple, $\Phi(r)=-\alpha / r$, with $\alpha$ a positive constant. Now, if $\bar{T}_{\mu}^{\nu}=\operatorname{diag}[\bar{\rho},-\bar{\tau}, \bar{p}, \bar{p}]$ (energy density, tension and pressure respectively), the nonvanishing components of the fields equations are

$$
\begin{gathered}
8 \pi \bar{\rho}=-\frac{\alpha^{2}}{r^{4}}\left(1-\frac{b(r)}{r}\right)+\frac{\alpha}{2} \frac{b^{\prime}(r)}{r^{3}}-\frac{\alpha}{2} \frac{b(r)}{r^{4}} \\
8 \pi \bar{\tau}=\left(1-\frac{b(r)}{r}\right)\left(-\frac{2 \alpha}{r^{3}}+\frac{\alpha^{2}}{r^{4}}+\left(\varphi^{\prime}\right)^{2}\right)-\frac{\alpha}{2} \frac{b^{\prime}(r)}{r^{3}}+\frac{\alpha}{2} \frac{b(r)}{r^{4}}-\frac{b^{\prime}(r)}{r^{2}}+\frac{b(r)}{r^{3}} \\
8 \pi \bar{p}=\frac{b^{\prime}(r)}{2 r^{2}}-\frac{\alpha}{r^{3}}\left(1-\frac{b(r)}{r}\right)+\frac{b(r)}{2 r^{3}} \\
\left(\varphi^{\prime}\right)^{2}=\kappa^{2} \frac{e^{2 \alpha / r}}{r^{3}[r-b(r)]}
\end{gathered}
$$

with $\kappa$ an integration constant. Imposing the traceless constraint we first obtain

$$
b^{\prime}(r)+b(r) \frac{2 \alpha^{2}-\alpha r}{r^{2}(\alpha+2 r)}-\frac{2 \alpha^{2}+\kappa^{2} e^{2 \alpha / r}}{r(\alpha+2 r)}=0 .
$$

After the change of variables, $x=2+\alpha / r$, Eq. (16) integrates straightforwardly to

$$
b(x)=\alpha\left(\frac{1}{x}+\frac{2}{x^{2}}+\frac{3}{x^{3}}+\frac{3}{x^{4}}+\frac{3}{2 x^{5}}-\frac{\kappa^{2}}{5 \alpha^{2}} e^{2(x-2)}+\frac{\mathcal{K}}{x^{5}} e^{2(x-2)}\right),
$$

where $\mathcal{K}$ is an integration constant to be determined.

Every wormhole (recall that we are dealing with MT's wormhole), by its definition, must have a minimum radius $r_{\text {th }}$ (the wormhole throat) at which its embedded surface is vertical, and consequently the radial coordinate is ill behaved. However, as usual one defines the proper radial distance

$$
l(r)= \pm \int \frac{d r}{[1-b(r) / r]^{1 / 2}}
$$


which is well behaved throughout the spacetime, i.e., $\forall r, 1-b(r) / r \geq 0$. For the space to be asymptotically flat, far from the throat we require that $b(r) / r \rightarrow 0$ as $l \rightarrow \pm \infty$. Notice that our shape function satisfies this condition irrespective of the choice of $\mathcal{K}$. In addition, the wormhole must flare outward near the throat. Stated mathematically, $b(r) / r \leq 1$, with the equality holding at the throat. This indeed constraints the value of $\mathcal{K}$ but not the absolute size of the throat which depends further on the parameter $\alpha$,

$$
\mathcal{K}=e^{-2\left(x_{\mathrm{th}}-2\right)} \frac{x_{\mathrm{th}}^{3}+3 x_{\mathrm{th}}^{2}+4.5 x_{\mathrm{th}}+3}{x_{\mathrm{th}}-2}+\frac{\kappa^{2} x_{\mathrm{th}}^{5}}{5 \alpha^{2}} .
$$

The aforementioned properties of $b(r)$, together with the condition on $\Phi$, entail that the metric tensor describes two asymptotically flat spacetimes joined by a throat. Notice that the solution goes over GR if $\varphi$ is equal to a constant [26].

We turn now to the analysis of the scalar field with two possible courses of cosmological evolution as background: i) The matter that threads the wormhole remains completely static decoupled from the cosmological expansion of the universe around. This regime actually cannot be completely correct since the asymptotic gravitational constant does evolve. However, in a more realistic situation one could just naïvely expect that the effect of $\phi$ at the throat varies much slower than the cosmological evolution of $G$. ii) The energy momentum tensor adjusts its parameters to the change of $G$ at short intervals of time when comparing with the scale of cosmological evolution.

At this stage it is worthwhile to remark that despite some kind of "evolution" is implicit between the matter and the scalar sectors, we are dealing with a static metric during the whole proccess. This means that the location of the extremal hypersurfaces which might see any in-coming and/or out-going null geodesic always coalesce in the center of the wormhole [7]. We are not going to discuss here about the "evolution" of the stress energy tensor nor the stability of the wormhole solution, being this beyond the scope of this letter. Instead we content ourselves analyzing snapshots of wormhole-like spacetimes. Afterwards, assuming that these configurations lay in the path of the real cosmological evolution, we are able to perform a preliminary research on gravitational memory of natural wormholes. 
Replacing Eq. (7) in Eq.(15) we obtain an ordinary differential equation for $\phi$ and $\omega$,

$$
\sqrt{\omega(\phi)+3 / 2} \frac{\phi^{\prime}}{\phi}=\kappa \frac{e^{\alpha / r}}{r^{2}} \frac{1}{[1-b(r) / r]^{1 / 2}} .
$$

Although there is no a priori prescription about the coupling function, to make a proper study of a varying gravitational strength it is imperative to operate within a self-consistent scenario. In this direction, cosmological solutions consistent with weak field limits were derived in Ref. [27]. This group (hereafter referred to as type I solutions) allows $\phi$ to evolve into asymptotic values consistent with current weak field limits independently of the imposed boundary condition, i.e., earlier values of $\phi_{\infty}$ may be equal to, less than, or bigger than 1. A second class of coupling functions may be described by a power-law dependence, $\omega(\phi) \propto \phi^{n}$ (type II solutions) [28]. In this case the parameter space is not constrained by weak field tests, but it is by nucleosynthesis processes $(n \geq 4$ is compatible with the observed abundance of primordial helium [29]). In order to solve Eq. (20) we had recourse to an adaptive stepsize fifth order Runge-Kutta method [30] setting $\alpha=r_{\mathrm{th}}=1$.

In the case of Brans Dicke theory, solutions were found independently of the value of $\omega$. Some examples are plotted in Fig. 1. As expected the solution becomes constant as $\omega \rightarrow \pm \infty$, i.e., the theory goes over GR. The slope of $\phi \rightarrow \infty$ as $r \rightarrow r_{\text {th }}$, but $\phi$ remains positive in all cases. However, within this framework solar system observations imply that the coupling constant need to be very large, actually, $|\omega|>500$ [24]. Thus, possible modifications to the wormhole parameters are negligible whether or not there is gravitational memory.

For type I solutions [27] we found that $\phi$ remains constant throughout the spacetime. Thus, assuming that in any wormhole geometry $\phi^{\prime \prime}(x)<0$ in the entire domain of $x$, to mantain the wormhole opened (within a hypothetical $G$ time-varying scenario) the matter sector must evolve adjusting its value to the ADM mass with the changing $G$ (for definition of ADM mass see, for instance, chapter 11 of Ref. [2]).

When the coupling function was assumed to be $2 \omega+3=\omega_{0}\left(\phi / \phi_{\infty}^{\text {today }}\right)^{n}$ [28] it became apparent that the existence of the solution is strongly correlated with the values of $\omega_{0}$ and 
$n$. In some cases the slope of the scalar field becomes infinite before reaching the throat (e.g. $\omega_{0}=1, n=1$ ), yielding no wormhole solutions. Nonetheless, well behaved solutions are attainable for bounded regions of the parameter space. In particular, we have plotted in Fig. 1 the one corresponding to $\omega_{0}=100, n=4$.

To give a step further we changed the asymptotic values for the scalar field and repeated the previous analysis (notice that because of the change in the boundary condition, regions of the parameter space which have shown singular solutions might acquire analyticity and vice versa). Our results are listed in Table I. As was expected, for type I solutions, if one considers the final stages of the cosmological evolution, the rate of change for the scalar at the throat is bigger than its asymptotic behavior. For instance, a change of $\phi_{\infty} \sim 10 \%$ yields a variation of $69 \%$ at the throat. Remarkably, at the very early universe the situation is turned over, i.e., a $10 \%$ in the variation of $\phi_{\infty}$ corresponds to a $5 \%$ in the variation of $\phi_{\mathrm{th}}$. For coupling "constants" (type II) compatible with nucleosynthesis tests, the rate of change of $\phi$ is similar at $x=2$ and $x=3$, whereas if, $2 \omega+3=10 \phi / \phi_{\infty}^{\text {today }}$, the variation at the wormhole throat may be slower than that at $r \rightarrow \infty$. All in all, contrary to type I, for type II solutions one could expect that the local value of $G$ within the wormhole might be preserved while its asymptotic value evolves with a cosmological rate. It would also be desirable a well behaved cosmological evolution of such $\omega(\phi)$. Unfortunately, one need to be aware that the last scheme is not consistent with nucleosynthesis tests. In any case, it is straightforward that gravitational memory phenomenon - if it is not forbidden by the laws of physics [31] - might provide a way of survival for natural wormholes.

\section{ACKNOWLEDGMENTS}

We are indebted to D. Monteoliva for computational help, and to D. F. Torres for critical reading of the draft. LAA wishes to thank the International Centre for Theoretical Physics at Trieste for kind hospitality during the preparation of the manuscript. This work has been partially supported by FOMEC (LAA), CONICET (MLT-HV), and UNLP (MLT-HV-FZ). 


\section{TABLES}

TABLE I. Relation between the scalar field at the throat and its asymptotic value.

\begin{tabular}{|c|c|c|}
\hline $2 \omega+3$ & $\phi_{\infty}$ & $\phi_{\mathrm{th}}$ \\
\hline \multicolumn{3}{|c|}{$10\left|1-\phi / \phi_{\infty}^{\text {today }}\right|^{-1 / 2}$} \\
\hline & 1 & 1 \\
\hline & .98 & .31 \\
\hline & .9 & .27 \\
\hline & .8 & .22 \\
\hline \multicolumn{3}{|c|}{$10^{4}\left(\phi / \phi_{\infty}^{\text {today }}\right)^{4}$} \\
\hline & 1 & .95 \\
\hline & .98 & .93 \\
\hline & .9 & .84 \\
\hline & .8 & .74 \\
\hline \multicolumn{3}{|c|}{$10^{5}\left(\phi / \phi_{\infty}^{\text {today }}\right)^{4}$} \\
\hline & 1 & .98 \\
\hline & .98 & .96 \\
\hline & .9 & .88 \\
\hline & .8 & .78 \\
\hline \multicolumn{3}{|c|}{$10 \phi / \phi_{\infty}^{\text {today }}$} \\
\hline & 1 & .06 \\
\hline & .9 & .04 \\
\hline
\end{tabular}




\section{REFERENCES}

[1] S. W. Hawking and G. F. R. Ellis, The Large Scale Structure of Space-Time, (Cambridge Univ. Press, Cambridge, 1973).

[2] M. Visser, Lorentzian Wormholes: from Einstein to Hawking, (American Institute of Physics, Woodbury, 1995).

[3] L. Flamm, Phys. Z. 17, 48 (1916).

[4] A. Einstein and N. Rosen, Phys. Rev. 48, 73 (1935).

[5] J. A. Wheeler, Phys. Rev. 97, 511 (1955).

[6] M. S. Morris and K. S. Thorne, Am. J. Phys. 56, 395 (1988).

[7] D. Hochberg and M. Visser, Phys. Rev. D 56, 4745 (1997); Phys. Rev. Lett. 81, 746 (1998); Phys. Rev. D 58, 044021 (1998).

[8] M. Visser, Phys. Rev D 56, 7578 (1997); Science 276, 88 (1997).

[9] The idea of wormholes as gravitational lenses was introduced in, J. G. Cramer, R. L. Forward, M. S. Morris, M. Visser, G. Benford and G. A. Landis Phys. Rev. D 51, 3117 (1995). A relation of negative mass microlensing effects with gamma ray bursts was put forward later, D. F. Torres, G. E. Romero and L. A. Anchordoqui, Mod. Phys. Lett. A 13, 1575 (1998); Phys. Rev. D 58, 123001 (1998).

[10] L. A. Anchordoqui, G. E. Romero, D. F. Torres and I. Andruchow, Mod. Phys. Lett. A 14, 791 (1999); G. E. Romero, D. F. Torres, I. Andruchow, L. A. Anchordoqui and B. Link, Mon. Not. R. Astron. Soc. (to be published), astro-ph/9904107.

[11] S. W. Hawking, Phys. Rev. D 46, 603 (1992).

[12] It is interesting to recall that a wormhole with the two mouths embedded in a single asymptotically flat universe and the time running at different rates on either side of the throat posses causal anomalies in the form of closed timelike curves. See, M. S. 
Morris, K. S. Thorne, U. Yurtserver, Phys. Rev. Lett. 61, 1446 (1988); V. P. Frolov, I. D. Novikov, Phys. Rev. D 42, 1057 (1990).

[13] D. Hochberg and T. Kephart, Phys. Rev. Lett. 70, 2665 (1993).

[14] D. Hochberg, Phys. Lett. B251, 349 (1990); K. Ghoroku and T. Soma, Phys. Rev. D 46, 1507 (1992).

[15] J. Moffat and T. Svodoba, Phys. Rev. D 44, 429 (1991).

[16] B. Bhawal and S. Kar, Phys. Rev. D 46, 2464 (1992).

[17] A. Agnese and M. La Camera, Phys. Rev. D 51, 2011 (1995); K. K. Nandi, A. Islam and J. Evans, Phys. Rev. D 552497 (1997); L. A. Anchordoqui, S. E. Perez Bergliaffa and D. F. Torres, Phys. Rev. D 55, 5226 (1997); K. K. Nandi, B. Bhattacharjee, S. M. K. Alam and J. Evans, Phys. Rev. D 57, 823 (1998); L. A. Anchordoqui, Nuovo Cimento 113B, 1497 (1998); L. A. Anchordoqui, G. Grunfeld, and D. F. Torres, Grav. Cosm. 4, 287 (1998). See also, P. E. Bloomfield, Phys. Rev. D 59, 088501 (1999); K. K. Nandi, Phys. Rev. D 59, 088502 (1999).

[18] P. G. Bergmann, Int. J. Theor. Phys. 1, 25 (1968); R. V. Wagoner, Phys. Rev. D 1, 3209 (1970); K. Nordvedt Jr., Astrophys. J. 161, 1059 (1970).

[19] D. F. Torres and H. Vucetich, Phys. Rev. D 54, 7373 (1996).

[20] D. La and P. J. Steinhardt, Phys. Rev. Lett. 62, 376 (1989).

[21] J. D. Barrow, Phys. Rev. D 46, 3227 (1992); Gen. Rel. Grav. 26, 1 (1994).

[22] D. F. Torres, A. Liddle and F. E. Schunck, Phys. Rev. D 57, 4821 (1998).

[23] It should be noticed that within this framework euclidean solutions, as well as microscopic charge-carrying Wheeler's wormholes have been previously found out. See, X-G. Xiao and L. Liu, Int. J. Theor. Phys. 35, 1503 (1996); T. Kodama, Phys. Rev. D 18, 3529 (1978); N. Van den Bergh, Gen. Rel. Grav. 15, 449 (1983). 
[24] C. M. Will, Theory and experiment in gravitational physics, (Cambridge Uni. Press, Cambridge 1981).

[25] R. M. Wald, General Relativity, (Univ. of Chicago Press; Chicago 1984).

[26] S. Kar and D. Sahdev, Phys. Rev. D 52, 2030 (1995).

[27] J. D. Barrow and P. Parsons, Phys. Rev. D 55, 1906 (1997).

[28] J. D. Barrow and J. P. Mimoso, Phys. Rev. D 50, 3746 (1994).

[29] D. F. Torres, Phys. Lett. B 359, 249 (1995).

[30] W. H. Press, S. A. Teukolsky, W. T. Vetterling and B. P. Flannery, Numerical Recipes in FORTRAN, 2nd ed. (Cambridge Univ. Press, Cambridge, England, 1992).

[31] A word of caution: Very recently results from perturbative analysis around a black hole horizon indicate that there may be no such gravitational memory. T. Jacobson, astro-ph/9905303. 


\section{FIGURES}

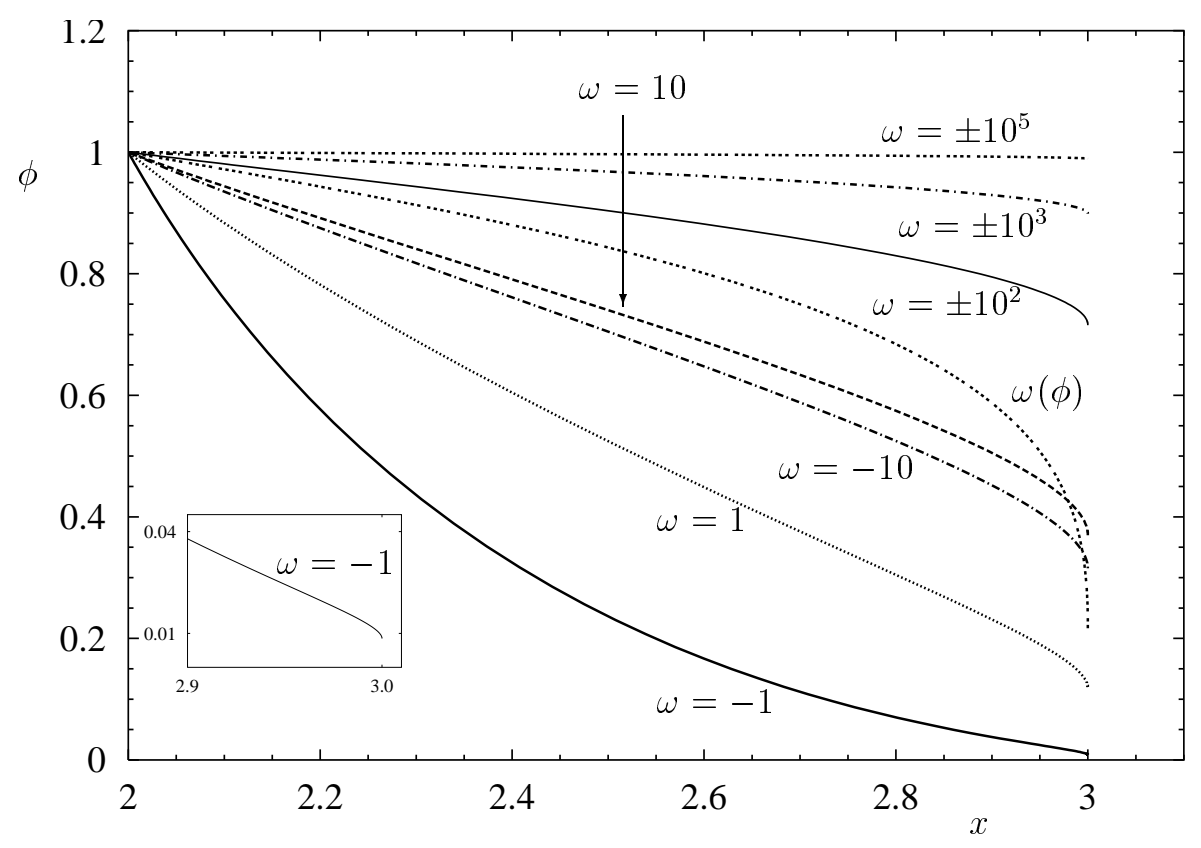

FIG. 1. Behavior of the scalar field as a function of the radial coordinate for typical models of the coupling constant $\omega(\phi)$. The embedded box details the behavior of $\phi$ when it gets close to the wormhole throat. 